\title{
The structure of NMDA receptor revealed
}

\section{Editorial}

N-Methyl-D-aspartate (NMDA) receptors are cation channels gated bytheneurotransmitter glutamate in the central nervous system. An NMDA receptor consists of three or four subunits from three subfamilies and seven different subunit types: the obligatory GluN1, four GluN2 (A-D), and two GluN3 (A-B) subunits. ${ }^{1}$ The activation of NMDA receptors by bindings of glutamate and glycine, together with postsynaptic depolarization, removes the $\mathrm{Mg}^{2+}$ that blocks the pore of NMDA receptor while cell is at rest and causes $\mathrm{Na}^{+}$and $\mathrm{K}^{+}$ ions to flow across the cell membrane through the pore. Furthermore, activated NMDA receptors also allow the entry of $\mathrm{Ca}^{2+}$ into the cell which will then trigger a series of cellular events, such as activations of $\mathrm{Ca}^{2+}$ calmodulin-depedent protein kinase (CaMKII), protein kinase $\mathrm{C}$ (PKC) and the insertion of another type of glutamate receptor, $\alpha$-amino-3-hydroxy-5-methyl-4-isoxazolepropionic acid (AMPA) receptor, into the postsynaptic membrane via exocytosis, ${ }^{2}$ so that the synaptic response to glutamate is strengthened. This is believed to be the basis of learning and memory. Dysfunctions of NMDA receptors may contribute to pain sensation and various brain disorders such as depression, schizophrenia, Huntington's disease, Alzheimer's disease, and Parkinson's disease. ${ }^{3}$

Recently, two independent studies, by Karakas \& Furukawa ${ }^{4}$ and Lee et al., ${ }^{5}$ revealed the first insight of the crystal structure of NMDA receptor in almost complete configurations. In both studies, the NMDA receptors are heterotetrameric complexes comprised of two GluN1 and two GluN2B subunits. In order to stabilize the crystal structures, both groups used ligands, agonists, or antagonists, together with introductions of multiple mutations in the subunits and eventually obtained crystals of NMDA receptor in an inactive state. Both structures (Protein Data Bank IDs: 4PE5 and 4TLL) have resolutions better than $4 \AA$, and both show two key characteristics reminiscent of the subunit organization of AMPA receptor obtained five years ago. ${ }^{6}$ First, the two GluN1 subunits and the two GluN2 subunits are arranged in an alternate pattern around the central pore, with twofold symmetry between the two GluN1-GluN2 heterodimers. Secondly, the receptor domains are layered. The amino terminal domain (ATD), which controls the gating of the channel and allows modulator bindings, is at the exterior end. The transmembrane domain (TMD), which provides the basis of the ion channel stays in the cell membrane. The ligand-binding domain (LBD), hosting the binding sites of ligands or their agonists and antagonists, is sandwiched between the ATD and the LBD. Going from the ATD to the LBD, the subunits in the two heterodimers swap domains, therefore the four subunits are intertwined into one complex. However, compared to the AMPA receptor structure, the ATD layer of NMDA receptor involves a much larger interaction interface therefore the ATD is more compactly packed within itself and against the LBD. Using engineered disulfide cross-links, Karakas and Furukawa further confirmed the physiological relevance of the distinct subunit interactions. The two groups also reached a consensus that the pore structure in the TMD shows a high similarity to potassium channels structure. ${ }^{7,8}$ Moreover,
Volume I Issue 2 - 2014

He Liu

Department of Biology, Gannon University, USA

Correspondence: He Liu, Department of Biology, Gannon University, Erie, PA 1654I,USA, Email liu017@gannon.edu

Received: October 03, 2014 | Published: October 06, 2014

Karakas and Furukawa used lanthanides to show the putative $\mathrm{Ca}^{2+}$ binding sites at the linker region connecting the LBD and the TMD. Lee et al. found a probable blocker binding site in the central vestibule to prevent ions from entering the selectivity filter. In summary, the structures identified by the two groups are highly similar, and the slight differences in crystal preparation and structural features complement one another. Although limited only to the inactive state, together these two studies provide a blueprint of the molecular structure of NMDA receptor and a framework for exploring the structures of other NMDA receptors with different compositions or in other states.

\section{Acknowledgements}

None.

\section{Conflict of interest}

The author declares no conflict of interest.

\section{References}

1. Hansen K, Ogden $\mathrm{K}$, Yuan $\mathrm{H}$, et al. Distinct Functional and Pharmacological Properties of Triheteromeric GluN1/GluN2A/GluN2B NMDA Receptors. Neuron. 2014;81(5):1084-1096.

2. $\mathrm{Lu} \mathrm{W,} \mathrm{Man} \mathrm{H}, \mathrm{Ju} \mathrm{W}$, et al. Activation of synaptic NMDA receptors induces membrane insertion of new AMPA receptors and LTP in cultured hippocampal neurons. Neuron. 2001;29(1):243-254.

3. Zhou Q, Sheng M. NMDA receptors in nervous system diseases. Neuropharmacology. 2013;74:69-75.

4. Karakas E, Furukawa H. Crystal structure of a heterotetrameric NMDA receptor ion channel. Science. 2014;344(6187):992-997.

5. Lee C, Lü W, Michel J, et al. NMDA receptor structures reveal subunit arrangement and pore architecture. Nature. 2014;511(7508):191-197.

6. Sobolevsky A, Rosconi M, Gouaux E. X-ray structure, symmetry and mechanism of an AMPA-subtype glutamate receptor. Nature. 2009;462(7274):745-756.

7. Doyle D, Morais Cabral J, Pfuetzner R, et al. The structure of the potassium channel: molecular basis of $\mathrm{K}^{+}$conduction and selectivity. Science. 1998;280(5360):69-77.

8. Zhou Y, Morais-Cabral J, Kaufman A, et al. Chemistry of ion coordination and hydration revealed by a $\mathrm{K}^{+}$channel-Fab complex at 2.0 A resolution. Nature. 2001;414(6859):43-48. 